04

\title{
Численное исследование возможности генерации убегающих электронов в формирующемся катодном слое самостоятельного объемного разряда высокого давления
}

\author{
(C) В.В. Лисенков ${ }^{1,2}$ \\ ${ }^{1}$ Институт электрофизики УрО РАН, \\ 620016 Екатеринбург, Россия \\ ${ }^{2}$ Уральский федеральный университет имени первого Президента России Б.Н. Ельцина, \\ 620083 Екатеринбург, Россия \\ e-mail: lisenkov@iep.uran.ru
}

Поступило в Редакцию 28 февраля 2019г.

В окончательной редакции 6 ноября 2019 г.

Принято к публикации 4 декабря 2019г.

\begin{abstract}
Проведены расчеты формирования катодного слоя самостоятельного объемного разряда высокого давления с предварительной ионизацией газовой среды, возбуждаемого нано- и субнаносекундными импульсами напряжения. Показано, что при давлениях $\sim 1 \mathrm{~atm}$ на завершающей стадии формирования катодного слоя создаются условия для генерации убегающих электронов. Рассмотрен также уход электронов в режим убегания из области усиления электрического поля перед фронтом плазменного (стримерного) канала, берущего начало из вершины микроострия на катоде. Показано, что при давлениях $\sim 10 \mathrm{~atm}$ создаются условия для убегания электронов сразу после их эмиссии с вершины микроострия в создаваемой им области усиления; полученные таким образом убегающие электроны сами могут создать предварительную ионизацию газовой среды и обеспечить формирование начальной фазы разряда в объемной форме в системах без подсветки.
\end{abstract}

Ключевые слова: убегающие электроны, объемный разряд, катодный слой.

DOI: $10.21883 / J T F .2020 .05 .49173 .70-19$

\section{Введение}

Исследование генерации убегающих электронов в газах высокого давления, в частности в воздухе, является одной из наиболее интересных задач физики газового разряда. Интерес к данному явлению обусловлен тем, что оно может иметь ряд практических приложений. Во-первых, это создание электронных ускорителей без фольги, разделяющей вакуумный и газовый объемы, которая является „слабым местом“ ускорителей. Вовторых, это получение электронных пучков пикосекундной длительности.

В целом состояние дел в данной области достаточно подробно описано в обзорах [1-3]. Более поздние результаты изложены в работах [4-7].

Известно, что уход электрона в режим убегания происходит при условии, когда среднее увеличение энергии электрона в электрическом поле становится больше, чем потери энергии в результате неупругих столкновений с молекулами газовой среды. Широко известен также аналитический критерий убегания электронов (см. [2] и цитируемую там литературу). Согласно данному критерию, убегание электронов реализуется при достаточно высоких напряженностях электрического поля, в разы превышающих пробивные значения. Поэтому генерация убегающих электронов реализуется в разрядах высокого давления, как правило, в отдельных локальных областях разряда, где на короткое время создаются необходимые условия. Именно этим обусловлен тот факт, что для получения пучков убегающих электронов обычно используются электродные системы с катодом специальной формы, обеспечивающие значительное усиление электрического поля в прикатодной области (см., например, $[4,8])$. Общеизвестной также является возможность появления убегающих электронов в области усиленного поля перед фронтом стримерного канала. Такая возможность была проанализирована, в частности, в обзоре [1] и книге [9], однако корректное численное моделирование методом Монте-Карло было проведено значительно позже $[2,3,10,11]$, причем большинство таких работ относится к исследованию генерации убегающих электронов во время формирования молнии $[2,10,11]$.

Известно также, что в катодном слое как тлеющего разряда низкого давления, так и объемного разряда высокого давления электрическое поле может многократно превышать среднее значение в разрядном промежутке даже при плоских электродах. Во многих случаях напряженность электрического поля формально достаточна для перехода электрона в режим убегания, однако такая возможность может далеко не всегда быть реализована из-за небольшого катодного падения напряжения (сотни вольт). В случае низкого давления даже такая небольшая энергия может быть достаточна для перехода электронов из высокоэнергетической части функции распределения 
по энергии в режим убегания. Такое явление также широко известно и неоднократно упоминалось в литеpaтуре (см., например, $[12,13])$. С увеличением давления возможность генерации убегающих электронов в катодном слое становится менее вероятной. Если для средних давлений генерация убегающих электронов еще упоминается в [14], то для высоких давлений такая генерация уже становится невозможной на квазистационарной стадии. Однако теоретические исследования процесса формирования катодного слоя [15-17] показали, что существует стадия, когда напряженность электрического поля становится уже достаточно высокой $\left(\sim 10^{5} \mathrm{~V} /(\mathrm{cm} \cdot \mathrm{atm})\right)$, а катодное падение еще не успевает снизиться до квазистационарных значений и остается на уровне киловольт. В таких условиях электроны могут уходить в режим убегания, и это было показано в [17] моделированием методом Монте-Карло. Однако используемая в вышеуказанных работах одномерная модель не в состоянии учесть всех особенностей данного процесса. В частности, с помощью одномерной модели невозможно посчитать убегание электронов перед фронтом плазменного (стримерного) канала, который может быть инициирован эмиссионной неоднородностью на поверхности катода, например микроострием. Усиление электрического поля перед фронтом такого канала в сочетании с усилением в формирующемся катодном слое может привести к более ранней генерации убегающих электронов по сравнению со значениями, полученными в одномерной модели.

Подобная ситуация ранее теоретически не исследовалась, несмотря на то что моделирование развития стримерного канала с катода было проведено в целом ряде работ. Это может быть обусловлено тем, что ранее исследователей интересовала контракция разряда, т.е. переход из объемной формы в искровую на заключительной стадии. Данное явление подробно описано в [9] и цитируемой там литературе. Кроме того, хотелось бы привести работу [18], не процитированную в [9].

Применительно к начальной стадии первые попытки $2 D$ моделирования были предприняты в работах $[19,20]$. В более поздней работе [21] было проведено более детальное $2 D$ моделирование развития стримерного канала с катода в формирующемся катодном слое. Было показано, что повышение эмиссии небольшого участка катода (природа которого не конкретизируется) ускоряет приближение фронта волны ионизации к катоду на этом участке по сравнению с соседними областями. Однако дальнейшее развитие неоднородности, вызванной этим пробоем, в работе [21] не рассматривается. В некотором смысле логическим продолжением данного исследования является работа [22]. В этой работе также с помощью $2 D$ модели рассматривается эволюция неоднородности, первоначально возникшей из небольшого участка плазмы около поверхности катода, обладающего более высокой проводимостью. Рассматривается динамика стягивания тока на этот участок и исследуется возможность дальнейшего формирования высокопроводящего искрового канала. Однако в вышеуказанных работах не рассматривается генерация убегающих электронов и их влияние на формирование самостоятельного объемного разряда.

Целью настоящей работы является численное исследование возможности и условий ухода электронов в режим убегания на этапе формирования катодного слоя самостоятельного объемного разряда с однородной предварительной ионизацией газовой среды.

\section{Описание модели и результаты расчетов}

Для проведения расчетов использовалась численная модель, которая базируется на системе уравнений для электронов, ионов, возбужденных атомов и уравнении Пуассона. Кинетические коэффициенты, зависящие от электрического поля, в том числе вероятность ухода электрона в режим убегания, были получены в результате моделирования движения электрона методом Монте-Карло. Подробное описание данной модели приведено в работах [17,23]. $1 D$ расчеты формирования объемного разряда с предварительной ионизацией газовой среды были проведены ранее в [15] для смеси $\mathrm{CO}_{2}: \mathrm{N}_{2}: \mathrm{He} \mathrm{c} \mathrm{давлением} 1 \mathrm{~atm}$ применительно к экспериментальным условиям [24,25], и в [17] для $\mathrm{N}_{2}$ с давлением $4 \mathrm{~atm}$ применительно к условиям [26-29]. В настоящей работе исследование будет проводиться на примере азота при давлении $1 \mathrm{~atm}$. В качестве начальных условий был взят предварительно однородно ионизованный разрядный промежуток длиной $0.5 \mathrm{~cm}$. На него подавался импульс напряжения амплитудой $50 \mathrm{kV}$ с линейным фронтом нарастания длительностью $50 \mathrm{~ns}$.

Результаты расчетов приведены на рис. 1. На нем представлены пространственные распределения концентрации электронов (рис. 1, $a$ ) и напряженности электрического поля (рис. $1, b)$ в различные моменты времени. Они иллюстрируют качественную картину формирования катодного слоя самостоятельного объемного разряда. Видно, что на начальной стадии, когда концентрация электронов в промежутке сравнительно мала, плазменный столб отодвигается от катода на $0.36 \mathrm{~cm}$. При этом концентрация электронов быстро нарастает. Между плазменным столбом и катодом образуется зона, обедненная зарядами, в которой концентрация ионов больше, чем электронов. Ионы частично экранируют внешнее поле, ослабляя его в плазменном столбе и усиливая в прикатодной области. В результате замедляется рост концентрации электронов в столбе и, как следствие, - рост плотности тока. Увеличение скорости ионизации в прикатодной области приводит к тому, что ионизационная волна (плазменный столб) быстро (31-32 ns) приближается к катоду, образуя катодное падение напряжения.

Характеристики формирующегося катодного слоя представлены на рис. 2. На рис. 2, a показаны изме- 

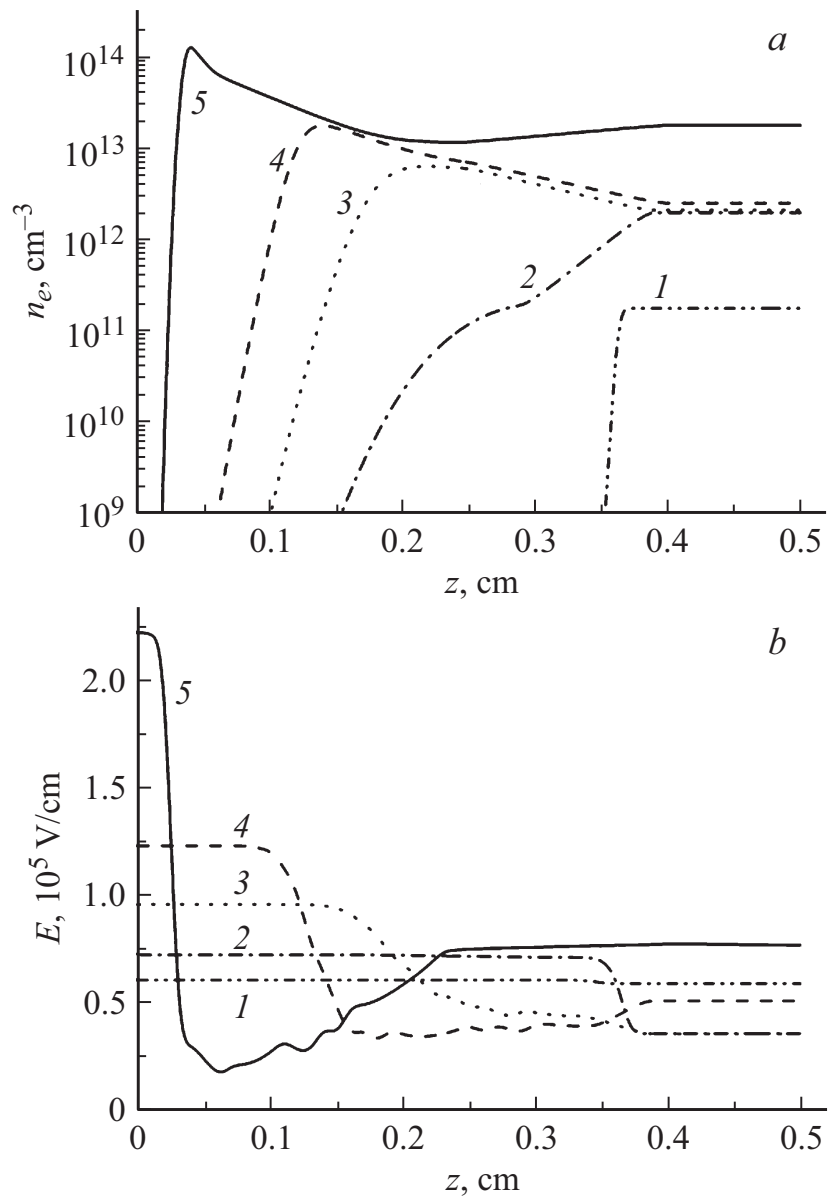

Рис. 1. Распределения концентрации электронов (a) и напряженности электрического поля $(b)$ в межэлектродном промежутке $(z-$ расстояние от катода) в различные моменты времени: $1-30,2-31,3-31.5,4-31.7,5-31.95 \mathrm{~ns}$.

нения во времени катодного падения напряжения $\left(U_{c}\right)$ и напряженности электрического поля $\left(E_{c}\right)$ около поверхности катода. На рис. $2, b$ показана соответственно динамика изменения протяженности катодного слоя $\left(d_{c}\right)$ и роста плотности тока $(j)$ в разрядном промежутке. Видно, что при приближении волны ионизации к катоду (уменьшении $d_{c}$ ) происходит увеличение напряженности электрического поля в катодном слое и уменьшение катодного падения напряжения. Последнее обстоятельство создает предпосылки к развитию неустойчивостей в катодном слое. Все это происходит при росте проводимости плазменного столба и увеличении плотности тока в разрядном промежутке.

Моделирование движения электрона методом Монте-Карло показало, что в промежутке времени 31.95-32.04 ns в рассчитанных выше условиях возможен уход электронов в режим убегания с их дальнейшим ускорением в разрядном промежутке, т. е. электрическое поле достаточно высокое для перехода в режим убегания, и катодное падение тоже еще достаточно для того, чтобы электрон набрал энергию, необходимую для продолжения режима убегания в более низком электрическом поле плазменного столба разряда.

Однако существует возможность более раннего появления убегающих электронов в формирующем катодном слое. Например, электрон, эмитированный с вершины микроострия, может инициировать развитие электронной лавины, которая, достигнув размера, близкого к критическому, может усилить электрическое поле перед своим фронтом и сделать возможным более чем в других областях формирующегося катодного слоя, раннее убегание электронов. Аналитическая модель, описывающая подобный механизм, изложена в [7,30], но непосредственное ее применение в настоящих расчетах невозможно, поскольку она использует ряд приближений, не выполняющихся в условиях настоящей работы и, как следствие, не позволяющих провести точный расчет. Поэтому в настоящей работе использована гибридная модель, представленная в [31]. В этой модели размножение электронов в области усиления электрического поля вблизи микроострия рассчитывалось моделированием их движения и столкновений методом Монте-Карло. После превышения числа электронов в лавине $\sim 10^{6}$



Рис. 2. Зависимость интегральных параметров формирующегося катодного слоя от времени. $a-$ катодное падение напряжения $\left(U_{c}\right)$, максимальная напряженность поля $\left(E_{c}\right)$; $b$ - протяженность катодного слоя $\left(d_{c}\right)$, плотность тока в разрядном промежутке $(j)$. 


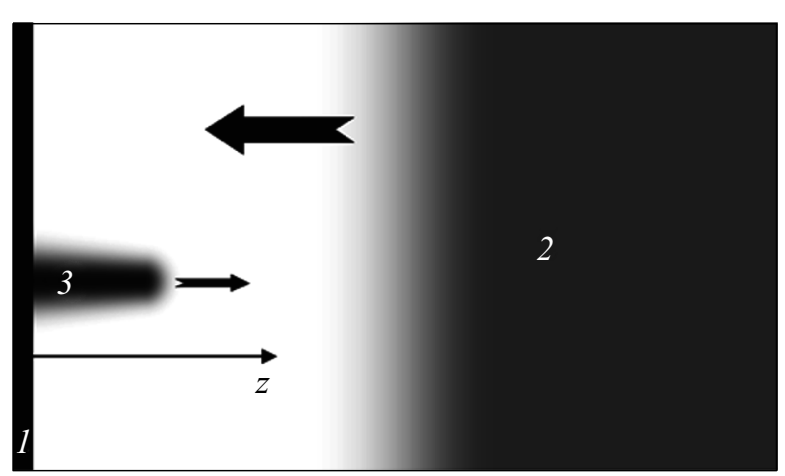

Рис. 3. Иллюстрация расчетной геометрии внутри катодного слоя. 1 - катод, 2 - плазменный столб, 3 - плазменный (стриммерный) канал, стартовавший из вершины микроострия на катоде. Оттенками серого условно показана степень ионизации участков межэлектродного объема. Стрелками показаны направление движения плазменного столба к катоду при формировании катодного слоя и направление движения канала навстречу плазменному столбу. Показано также направление оси $z$ для рис. 4 .

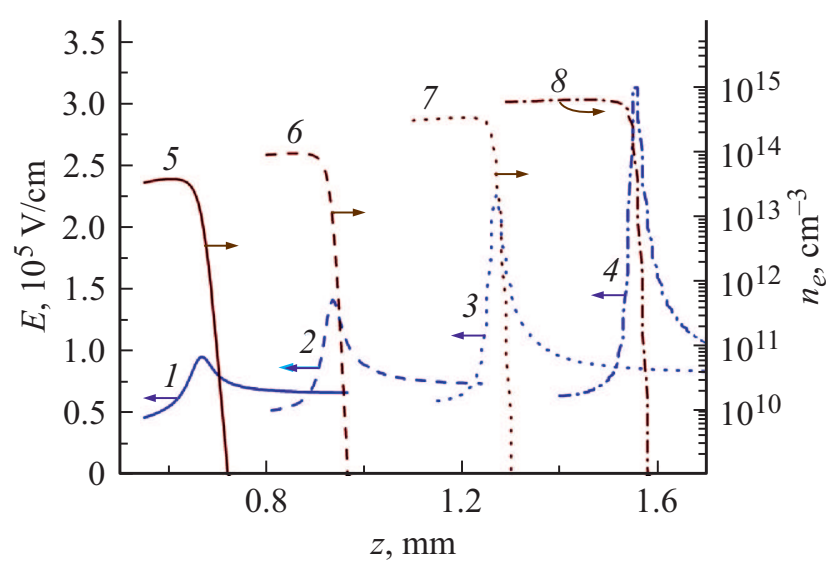

Рис. 4. Динамика измерения волнового фронта плазменного канала, инициированного лавиной, стартовавшей с микроострия высотой $10 \mu \mathrm{m}$ в момент времени $28 \mathrm{~ns}$ в формирующемся катодном слое в азоте для условий, соответствующих рис. 1,2 . Кривые 1-4 - концентрация электронов, кривые 5-8напряженность электрического поля. Кривые 1 и 5 соответствуют моменту времени $30.1 \mathrm{~ns}, 2$ и $6-30.4 \mathrm{~ns}, 3$ и $7-$ $30.6 \mathrm{~ns}, 4$ и $8-30.7 \mathrm{~ns}$.

происходил переход к двумерному варианту гидродинамической модели, использованной в представленных выше $1 D$ расчетах. Как показали расчеты, электронная лавина, стартовавшая с микроострия, имеет вначале более компактные размеры и высокую концентрацию заряда по сравнению с лавиной, стартовавшей с плоской поверхности катода. Развиваясь, такая лавина способна вызвать искажения электрического поля внутри формирующегося катодного слоя самостоятельного разряда. В результате генерация убегающих электронов может начаться в усиленном поле перед фронтом такой лавины еще до того момента, когда в основном объеме созда- дутся такие условия. Расчетная геометрия представлена на рис. 3.

Результаты расчетов движения волнового фронта стримерного канала с катода в направлении плазменного столба, динамики изменения концентрации электронов и электрического поля представлены на рис. 4. Видно, что в области усиленного поля перед фронтом плазменного канала к моменту времени $30.7 \mathrm{~ns}$ создаются условия для генерации убегающих электронов, тогда как в основном объеме формирующегося катодного слоя в объемном разряде в азоте при $p=1 \mathrm{~atm}$ (рис. 1,2 ) такие условия еще не достигнуты. При этом лавина, инициировавшая плазменный канал, стартовала с микроострия высотой $h=10 \mu \mathrm{m}$ в формирующемся катодном слое в объемном разряде в азоте при $p=1 \mathrm{~atm}$ в момент времени $28 \mathrm{~ns}$ (ситуация соответствует рис. 1,2). Расчеты показали, что генерация убегающих электронов в данных условиях возможна только с плазменных каналов, стартовавших в диапазоне времени 25-31 ns. В каналах, стартовавших раньше, усиления электрического поля на фронте будет не достаточно для генерации убегающих электронов, а в стартовавших позже электроны, уходящие в режим

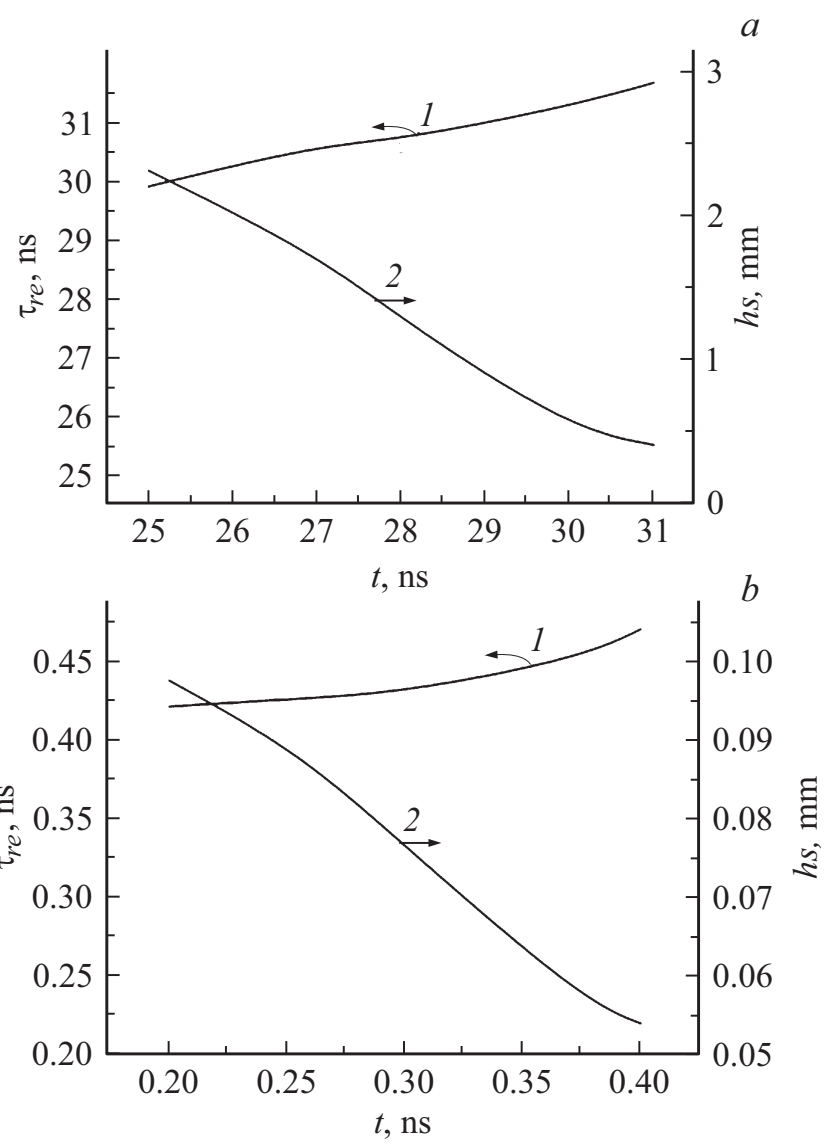

Рис. 5. Зависимость времени $\left(\tau_{r e}\right)$ (кривая 1) и высоты плазменного канала $(h s)$ (кривая 2), соответствующих началу режима убегания в области усиленного поля перед фронтом канала от момента старта канала с микроострия при формировании катодного слоя в объемном разряде в азоте при давлениях $1(a)$ и $10 \mathrm{~atm}(b)$. 
убегания, не успеют набрать достаточно энергии, чтобы продолжить убегание в плазменном столбе. При этом интервал времени, в котором возможен старт режима убегания, получается достаточно узким, всего $1.5 \mathrm{~ns}$ (рис. 5,a). Если смоделировать полностью геометрически подобную ситуацию при давлении $10 \mathrm{~atm}$, т.е. взять высоту микроострия $h=1 \mu \mathrm{m}$, то наблюдается полностью аналогичная ситуация, только процессы сдвигаются в субнаносекундный диапазон (рис. $5, b$ ). Однако для микроострия высотой $10 \mu \mathrm{m}$ ситуация меняется. В работе [32] показано, что при давлениях выше $10 \mathrm{~atm}$ электроны могут уходить в режим убегания непосредственно с вершины микроострия при напряженности электрического поля в межэлектродном промежутке, равной $820 \mathrm{kV} / \mathrm{cm}$. В работе [31] показано, что такая напряженность поля может реализовываться в самом начале формирования катодного слоя, что может дать старт анодонаправленому стримерному каналу, приводящему к контракции объемного разряда. С другой стороны, при отсутствии предварительной ионизации убегающие электроны могут ее обеспечить (правда, неоднородно) и привести к зажиганию разряда на короткое время в объемной форме перед его контракцией. Фото подобной стадии разряда приведено в [28].

\section{Заключение}

Проведенные расчеты показали возможность генерации убегающих электронов при формировании катодного слоя самостоятельного объемного разряда высокого давления. В рамках одномерной модели показана возможность ухода электронов в режим убегания на завершающем этапе формирования катодного слоя при давлениях $\sim 1 \mathrm{~atm}$. Применение двумерной гибридной модели позволило внести существенные уточнения и показать, что электроны могут уходить в режим убегания из области усиления электрического поля перед фронтом плазменного канала, берущего начало из вершины микроострия на катоде. При этом генерация убегающих электронов возможна и на более ранней стадии формирования катодного слоя.

В настоящей и более ранних работах показано, что при давлениях $\sim 10 \mathrm{~atm}$ может работать другой механизм ухода электронов в режим убегания. При таких давлениях и соответственно более высоких абсолютных значениях напряженности электрического поля создаются условия для ухода электронов сразу после их эмиссии с вершины микроострия в создаваемой им области усиления. Полученные таким образом убегающие электроны сами могут создать предварительную ионизацию газовой среды.

\section{Конфликт интересов}

Автор заявляет, что у него нет конфликта интересов.

\section{Список литературы}

[1] Бабич Л.П., Лойко Т.В., Цукерман В.А. // УФН. 1990. T. 160. № 7. C. 49-82. [Babich L.P., Loiko T.V., Tsukerman V.A. // Sov. Phys. Usp. 1990. Vol. 33. P. 521-540.]

[2] Гуревич А.В., Зыбин К.П. // УФН. 2004. Т. 171. № 11. C. 1177-1199. [Gurevich A.V., Zybin K.P. // Phys. Usp. 2001. Vol. 44. P. 1119-1140.] DOI: 10.1070/PU2001v044n11ABEH000939

[3] Тарасенко В.Ф., Яковленко С.И. // УФН. 2004. Т. 174. № 9. C. 953-971. [Tarasenko V.F., Yakovlenko S.I. // Phys. Usp. 2004. Vol. 47. P. 887-905.] DOI: 10.1070/PU2004v047n09ABEH001790

[4] Месяи, Г.А., Яландин М.И. и др. // Физика плазмы. 2012. T. 38. № 1. C. 34-51.[Mesyats G.A., Yalandin M.I. et.al. // Plasma Phys. Rep. 2012. Vol. 38. P. 29-45.] DOI: $10.1134 / \mathrm{S} 1063780 \mathrm{X} 11110055$

[5] Runaway Electrons Preionized Diffuse Discharges / Ed. by V. Tarasenko. USA: Nova Science, 2014. 613 p.

[6] Генерация убегающих электронов и рентгеновского излучения в разрядах повышенного давления / Под ред. В.Ф. Тарасенко. Томск: STT, 2015. 568 с. [Generation of Runaway Electron Beam and X-Ray in High Pressure Gases / Ed. by V. Tarasenko. USA: Nova Science, 2016. Vol. 1. 382 p. Vol. 2. 300 p.]

[7] Zubarev N.M., Yalandin M.I., Mesyats G.A. et. al. // J. Phys. D: Appl. Phys. 2018. Vol. 51. P. 284003.

[8] Костыря И.Д., Бакшт Е.Х., Тарасенко В.Ф. // ПТЭ. 2010. № 4. C. 84-87. [Kostyrya I.D., Baksht E.Kh., Tarasenko V.F. // Instrument. Experiment. Tech. 2010. Vol. 53. P. 545-548.]

[9] Королев Ю.Д., Месяи, Г.А. Физика импульсного пробоя газов. М.: Наука, 1991. 224 c. [Korolev Yu.D., Mesyats G.A. Physics of Pulsed Breakdown in Gases. Yekaterinburg: UD RAS, 1998. 275 p.]

[10] Chanrion O., Neubert T. // J. Geophys. Res. 2010. Vol. 115. P. A00E32. DOI: 10.1029/2009JA014774

[11] Celestin S., Pasko V.P. // J. Geophys. Res. 2011. Vol. 116. P. A03315. DOI: $10.1029 / 2010 J A 016260$

[12] Djakov B.E. // J. Phys. D: Appl. Phys. 1989. Vol. 22. P. 368 370.

[13] Kolobov V.I., Tsendin L.D. // Phys. Rev. A. 1992. Vol. 46. P. 7837-7852.

[14] Ткачев А.Н., Яковленко С.И. // ЖТФ. 2003. Т. 73. Вып. 2. C. 56-64. [Tkachev A.N., Yakovlenko S.I. // Tech. Phys. 2003. Vol. 48. P. 190-198.] DOI: $10.1134 / 1.1553559$

[15] Осипов В.В., Лисенков В.В. // ЖТФ. 2000. Т. 70. Вып. 10. C. 27-33. [Osipov V.V., Lisenkov V.V. // Tech. Phys. 2000. Vol. 45. P. 1258-1264.] DOI: 10.1134/1.1318960

[16] Осипов В.В. // УФН. 2000. Т. 170. № 3. С. 225-245. [Osipov V.V. // Phys. Usp. 2000. Vol. 43. P. 221-258.] DOI: $10.3367 /$ UFNr.0170.200003a.0225

[17] Иванов С.Н., Лисенков В.В. // ЖТФ. 2010. Т. 80. Вып. 1. C. 54-58. [Ivanov S.N., Lisenkov V.V. // Tech. Phys. 2010. Vol. 55. P. 53-57.] DOI: 10.1134/S1063784210010093

[18] Константинов М.Д., Осипов В.В., Суслов А.И. // ЖТФ. 1990. Т. 60. Вып. 10. С. 27-36.

[19] Simon G., Botticher W. // J. Appl. Phys. 1994. Vol. 76. P. 5036-5046. DOI: $10.1063 / 1.357215$

[20] Akashi H., Sakai Y., Tagashira H. // Aust. J. Phys. 1997. Vol. 50. P. 655-669. 
[21] Cernak M., Bessieres D., Paillol J. // J. Appl. Phys. 2011. Vol. 110. P. 053303. DOI: 10.1063/1.3630015

[22] Бычков Ю.И., Ямпольская С.А., Ястремский А.Г. // Изв. вузов. Физика. 2012. Т. 55. № 5. С. 3-12. [Bychkov Y.I., Yampolskaya S.A., Yastremskii A.G. // Russ. Phys. J. 2012. Vol. 55. P. 477-487.] DOI: $10.1007 / \mathrm{s} 11182-012-9837-\mathrm{y}$

[23] Лисенков В.В., Осипов В.В. // ЖТФ. 2007. Т. 77. Вып. 11. C. 49-54. [Lisenkov V.V., Osipov V.V. // Tech. Phys. 2007. Vol. 52. P. 1439-1444.] DOI: 10.1134/S106378420

[24] Бычков Ю.И., Осипов В.В. и др. // Изв. вузов. сер. Физика. 1986. N 4. C. 89-94.

[25] Мартынов И.П., Мехряков В.Н., Осипов В.В. // ЖТФ. 1992. Т. 62. Вып. 3. С. 58-62.

[26] Ivanov S.N., Shpak V.G. // IEEE Trans. Plasma Sci. 2011. Vol. 39. P. 2596-2597. DOI: 10.1109/TPS.2011.2157173

[27] Иванов С.Н. // ДАН. 2004. Т. 339. № 4. С. 472-476. [Ivanov S.N. // Dokl. Phys. 2004. Vol. 49. N 12. P. 701-705.] DOI: $10.1134 / 1.1848620$

[28] Ivanov S.N., Lisenkov V.V. // J. Appl. Phys. 2018. Vol. 124. P. 103304. DOI: $10.1063 / 1.5024974$

[29] Иванов С.Н., Лисенков В.В. // Физика плазмы. 2018. Т. 44. № 3. C. 323-332. [Ivanov S.N., Lisenkov V.V. // Plasma Phys. Reports. 2018. Vol. 44. P. 369-377.] DOI: $10.1134 / \mathrm{S} 1063780 \mathrm{X} 18030042$

[30] Зубарев Н.М., Иванов С.Н. // Физика плазмы. 2018. Т. 44. № 4. C. 397-406. [Zubarev N.M., Ivanov S.N. // Plasma Phys. Reports. 2018. Vol. 44. P. 445-452.] DOI: $10.1134 / \mathrm{S} 1063780 \mathrm{X} 18040104$

[31] Lisenkov V.V., Mamontov Yu.I. // J. Phys.: Conf. Series. 2018. Vol. 1141. P. 012051.

DOI: $10.1088 / 1742-6596 / 1141 / 1 / 012051$

[32] Лисенков В.В., Иванов С.Н., Мамонтов Ю.И., Тихонов И.Н. // ЖТФ. 2018. Т. 88. Вып. 12. С. 1912 1915. [Lisenkov V.V., Ivanov S.N., Mamontov Yu.I., Tikhonov I.N. // Tech. Phys. 2018. Vol. 63. N 12. P. 18721875.] DOI: $10.1134 / \mathrm{S} 1063784218120095$ 